This item was submitted to Loughborough's Research Repository by the author.

Items in Figshare are protected by copyright, with all rights reserved, unless otherwise indicated.

\title{
Evaluation of vibrotactile sensations in the 'feel' of a golf shot
}

PLEASE CITE THE PUBLISHED VERSION

http://dx.doi.org/10.1016/j.jsv.2004.08.030

\section{PUBLISHER}

(c) Elsevier Ltd.

\section{VERSION}

AM (Accepted Manuscript)

LICENCE

CC BY-NC-ND 4.0

\section{REPOSITORY RECORD}

Roberts, Jonathan R., Roy Jones, Neil J. Mansfield, and Steve Rothberg. 2019. "Evaluation of Vibrotactile Sensations in the 'feel' of a Golf Shot". figshare. https://hdl.handle.net/2134/11474. 
This item was submitted to Loughborough's Institutional Repository (https://dspace.lboro.ac.uk/) by the author and is made available under the following Creative Commons Licence conditions.

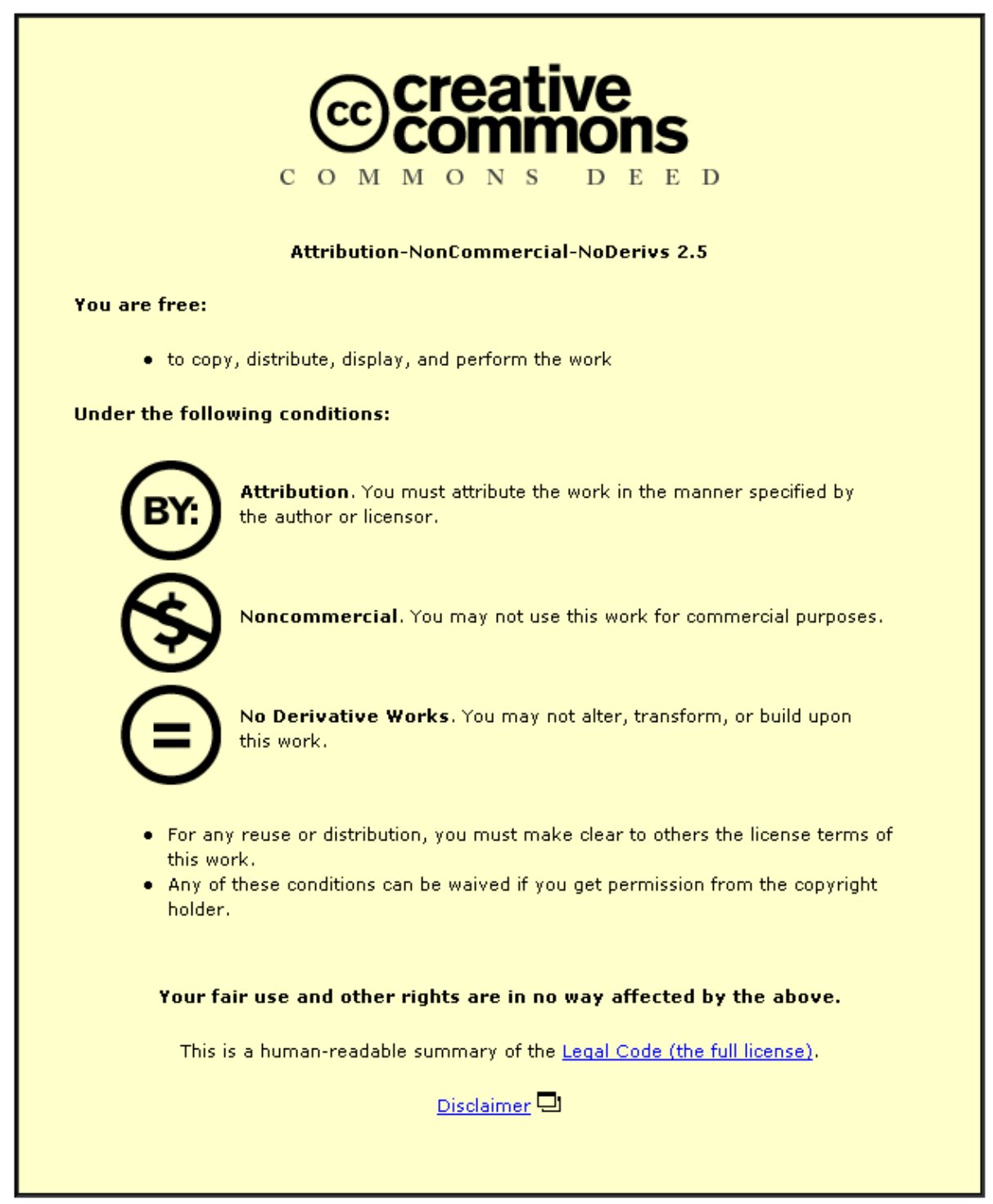

For the full text of this licence, please go to: http://creativecommons.org/licenses/by-nc-nd/2.5/ 


\title{
Evaluation of vibrotactile sensations in the 'feel' of a golf shot
}

\author{
J.R. Robets ${ }^{\mathrm{a}}$, R. Jones ${ }^{\mathrm{a}}$, N.J. Mansfield ${ }^{\mathrm{b}}$, S.J.Rothberg ${ }^{\mathrm{a}}$ \\ ${ }^{a}$ Wolfon School of Mechanical and Manufacturing Engineering, Loughborough University, Loughborough, \\ Leicestershire, LE11 3TU, UK \\ ${ }^{\mathrm{b}}$ Department of Human Sciences, Loughborough University, Loughborough, Leicestershire, LE11 3TU, UK
}

\begin{abstract}
Players' subjective perceptions of the characteristics, suitability and quality of sports equipment will have a significant bearing on their equipment selection. The 'feel' of a golf club is such a perception and the vibration at impact perceived by the player is generally considered to contribute significantly to 'feel'. The aim of this study was to investigate the correlation between golfers' subjective perceptions of the feel of a shot and the post-impact vibration of a club. Suitable test procedures were developed to quantify the golfers' perceptions and to obtain measurements of vibration at the hands. The five feel characteristics investigated, 'pleasantness', 'hardness', 'solidity', 'liveliness' and perceived vibration level, were found to be strongly correlated; shots that were regarded as having a pleasant feel were also rated as having felt solid, lively, soft and with little vibration perceived. When these ratings were correlated with raw measurements of impact vibration, the relationships were initially weak. Techniques, such as normalizing the data, weighting the data and using mean data, were developed resulting in much stronger correlations. Ultimately, a reduction in the total r.m.s. vibration level was found to correlate well with the players' subjective descriptions of 'pleasant', 'solid', 'lively' and 'soft' feel.
\end{abstract}

\section{Introduction}

In golf, players often use the word 'feel' to describe the feedback received from a number of internal and external sources. For example, a golfer will judge the quality of a swing by comparing the internal kinaesthetic feedback received from the limb movements that form the swing with the memory of previous swing experiences. External sources that stimulate either the auditory, tactile or visual systems during the swing, during the impact or postimpact will also influence the feel of a shot [1-3]. Feel is, therefore, associated with many different perceptions of a shot and as a result is very difficult to both define and quantify. This study concentrates on perceptions based on external sources of feedback because they are associated with the equipment used. Clearly the performance of a club is the main priority, especially with drivers, but the feel of a club is acknowledged as a significant factor when players select from a range of clubs with broadly similar performance.

The overall aim of this study was to identify properties of the vibration from impact responsible for different feel characteristics. To achieve this, test procedures were developed to quantify perceptions under play conditions, vibration measurements were selected to be representative of those perceived by the players and methods to correlate the subjective and objective data were formulated. Success will ultimately allow the feel of a golf club to be manipulated and improved at an early stage in the design process.

\subsection{Modal analysis of sports equipment}

Sound and vibration due to impact in golf are two of the predominant forms of external feedback received by the player. The vibration characteristics of sports equipment have been investigated to a greater extent than the sound characteristics, mainly because vibration at impact has been linked to injury, such as lateral epicondylitis (tennis elbow) $[4,5]$, and to discomfort or annoyance, particularly when the impact is not located at the 'sweet spot'.

Modal analyses of golf clubs have investigated the association between the 'sweet spot' and the location of nodal lines on the clubface $[6,7]$. Test conditions, however, are generally far removed from actual play conditions; the club is stationary and the boundary conditions are not always representative of a human grip. Modal analysis certainly has a role to play in predicting 'feel' and it has been found, for example, that the natural frequencies measured using a free-free condition do show good agreement with those obtained using a handheldfree condition [8]. However, dynamic influences during an actual swing, such as centrifugal stiffening of the shaft [9] or changes in grip condition [10, 11], have yet to be 
addressed and this requires measurement during play, as performed in this study.

1.2 Transmission of vibration to the hand and arm in sport

Many studies of the vibration of sports equipment have measured vibration of the implement itself rather than the vibration transmitted to the player. The vibration has rarely been measured at the grip location and so, in golf, it has not taken into account the complex mechanical coupling between the shaft, the rubber grip and the hand. Studies in tennis have assessed the injury potential of vibration transmission to the player by recording measurements at bone protrusions on the hand and arm $[4,5]$. Human skin attenuates vibration at higher frequencies and only low frequencies are transmitted into the bone structure [12]; the sensors in the hand, which are located under the surface of the skin, however, will respond to frequencies up to at least $1 \mathrm{kHz}$ [13] so such measurements will not represent the vibration perceived by the golfer. In this paper, measurement is at the handgrip interface.

\subsection{Subjective perception to vibration}

To date, few studies have combined vibration measurements with players' perceptions. Merkel and Blough [7] measured vibration from golf shots and obtained players' ratings of the feel and flight of the ball as either 'good' or 'bad'. The authors concluded that, for a 'good' hit, low frequency bending modes of the shaft dominate, whereas for a 'bad' hit, the majority of the energy is in higher frequency modes from 300 to $3000 \mathrm{~Hz}$. Other researchers, however, have demonstrated that a player's assessment of feel is much more complex than just 'good' or 'bad'. Hocknell et al. [3] constructed a ranking of the 'hardness' of feel for combinations of different club and ball types from the opinions of golfers using the equipment and from responses to a questionnaire. The authors concluded that a softer, more desirable sensation could be achieved by exciting modes of vibration from 500 to $2500 \mathrm{~Hz}$ more strongly than modes in the region of $100 \mathrm{~Hz}$. In this paper, the subjective perceptions are recorded from each player after every shot, with the objective data measured simultaneously.

\subsection{Human response to vibration}

Fundamental studies have generated threshold of perception and equal sensation contours to investigate sensitivity to hand-transmitted vibration [13, 14]. Typically, these studies have found that humans are most sensitive to frequencies in the range 50 to $300 \mathrm{~Hz}$. As frequency increases, sensitivity decreases; at $1 \mathrm{kHz}$, r.m.s. vibration accelerations approximately 100 times greater than at $100 \mathrm{~Hz}$ are required to produce an equivalent sensation. In previous sports equipment research, however, only a few studies have acknowledged the dependence of human sensitivity on vibration frequency $[3,15,16]$. In this paper, the suitability of standard frequency weightings, derived from subjective and biodynamic studies of the hand and arm, are investigated.

\section{Test methodology}

During a previous study, interviews were conducted with elite golfers to elicit their perceptions of the feel of shots hit with a number of different clubs and balls [17]. Ten general dimensions related to the feel of a golf shot emerged during the analysis. The tests also elicited the vocabulary used by the players enabling future questions to be phrased using familiar terminology.

\subsection{Selection of feel characteristics}

One of the emergent dimensions, 'Feel from Impact', was selected for further analysis as the themes grouped together within this dimension were thought to relate to the sound or the vibration from impact. During the interviews, the golfers generally described off-centre impacts as feeling 'hard', and the vibration level was perceived to be greater. In contrast, central impacts were described as 'soft' or 'sweet', and the golfers described feeling little if any vibration. The type of club also had an influence, with the older, wooden clubheads described as having a 'softer' feel than the modern, metal clubheads and the type of ball also influenced how 'hard' or 'soft' a shot felt. Club vibration from impact was, therefore, thought to contribute to the hardness of feel but, as the sound of the impact may also influence this characteristic, it was decided to measure the golfers' perceptions of vibration level and hardness of feel separately.

Golfers regularly described the feeling of the ball compressing and recovering during impact. With some clubs, typically traditional wooden headed clubs, golfers described feeling the ball being 'absorbed' by the clubhead and coming off slowly resulting in a 'dead' or 'dull' feel. In contrast, a more 'powerful' feel resulted when a ball 'exploded' or 'came quickly' off the clubface. This was also selected as a feel characteristic to be measured. Golfers frequently described shots as feeling 'solid', so this characteristic was selected, and, finally, it was decided to obtain an overall rating of the pleasantness of feel. For many of these characteristics, the contribution of sound to the golfers' perceptions may be important, so steps were taken during the test to isolate the sensation of club vibration as the only feedback received by the golfer.

\subsection{Measurement of subjective data}

In sport, two previous studies using scaled response questions have been reported in literature. In baseball, Noble and Walker [15] measured perceived discomfort using the category scale 'none', 'slight', 'moderate' and 'severe' whilst, in tennis, Stroede et al. [16] used a visual analogue scale, labelled 'comfortable on impact' and 'uncomfortable on impact' at each extreme. 
Table 1. Questions used to quantify golfers' perceptions of five feed characteristics

\begin{tabular}{|c|c|c|c|c|c|c|c|c|c|c|}
\hline \multicolumn{11}{|c|}{ How did the shot feel? } \\
\hline Unpleasant & 1 & 2 & 3 & 4 & 5 & 6 & 7 & 8 & 9 & Pleasant \\
\hline \multicolumn{11}{|c|}{ How did the impact feel in your hands? } \\
\hline Very soft & 1 & 2 & 3 & 4 & 5 & 6 & 7 & 8 & 9 & Very hard \\
\hline \multicolumn{11}{|c|}{ How much vibration did you feel in your hands? } \\
\hline No vibration & 1 & 2 & 3 & 4 & 5 & 6 & 7 & 8 & 9 & Lots of vibration \\
\hline \multicolumn{11}{|c|}{ How solid did the shot feel? } \\
\hline Not solid & 1 & 2 & 3 & 4 & 5 & 6 & 7 & 8 & 9 & Very solid \\
\hline \multicolumn{11}{|c|}{ How quickly did you perceive the ball to have come off the clubface? } \\
\hline $\begin{array}{c}\text { Dead, ball came } \\
\text { off slowly }\end{array}$ & 1 & 2 & 3 & 4 & 5 & 6 & 7 & 8 & 9 & $\begin{array}{c}\text { Lively, ball came } \\
\text { off quickly }\end{array}$ \\
\hline
\end{tabular}

In this study, scaled response questions were also used to quantify the golfers' perceptions. For each feel characteristic selected, a question was phrased and the golfer given a choice of responses from 1 to 9 with descriptive words used to give the scale orientation. The five questions used are listed in Table 1; in order to follow the analysis of the results, familiarity with the orientation of each scale is essential.

One problem with questions of this nature is that participants will use different ranges within the overall scale, especially early in the test when their reference levels are developing. To minimise this problem, the results from the first club were removed from the analysis and club order was randomised. Statistical techniques to overcome the variations in each golfer's reference level and their use of the scales were also investigated and are discussed further during the analysis of the results.

\subsection{Measurement of vibration data}

Based on standards for assessing injury potential [18, 19], the adapter in Figure 1a was developed on which two low profile accelerometers were mounted. The players confirmed that it could be comfortably gripped between the hand and the golf club during stroke play. The $80 \mathrm{~mm}$

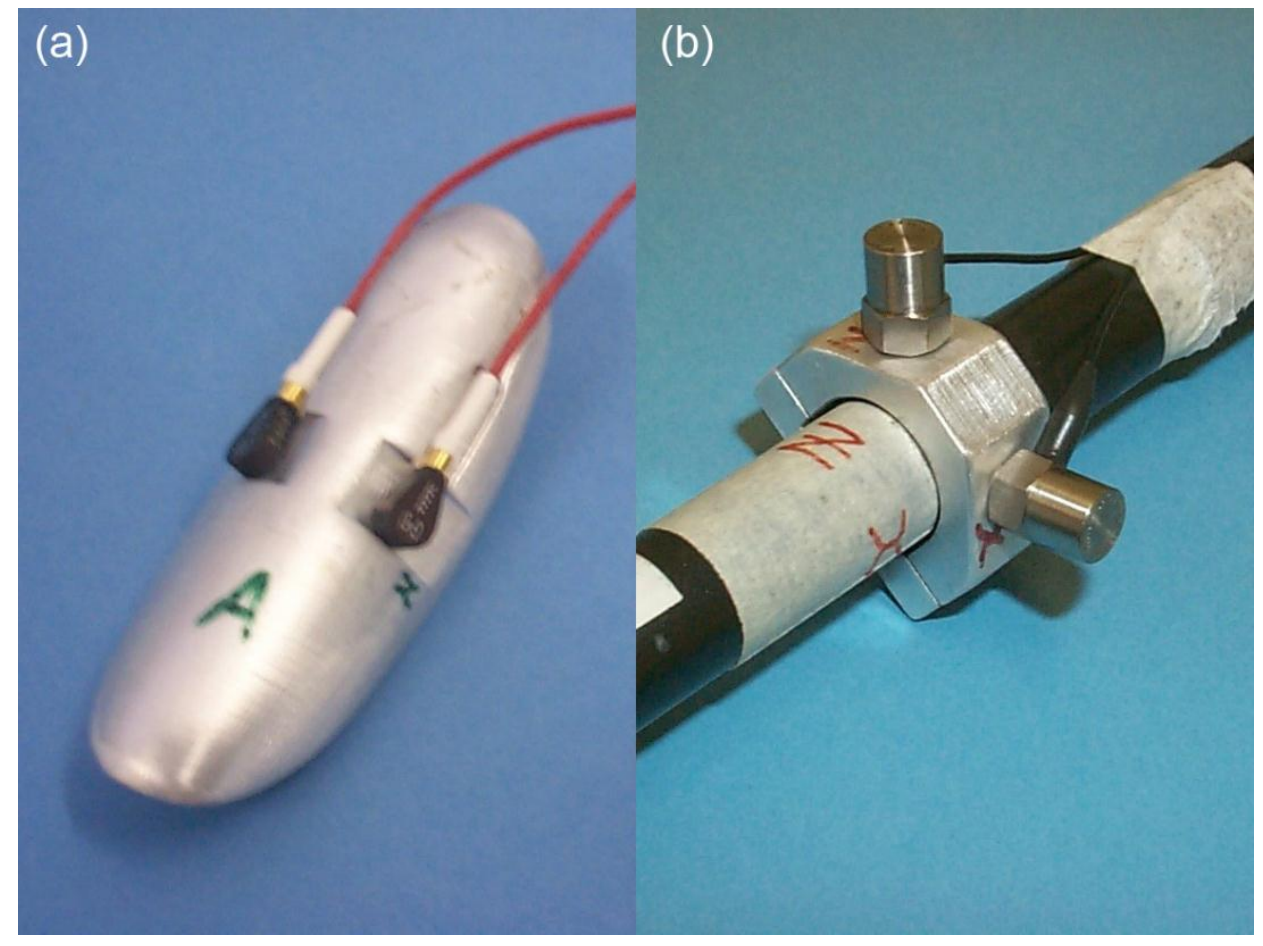

Figure 1. Accelerometer mounting brackets for measurement of vibration at (a) the hand-grip interface and (b) the shaft. 
a) Frequency response of the adaptor in the $x$-direction
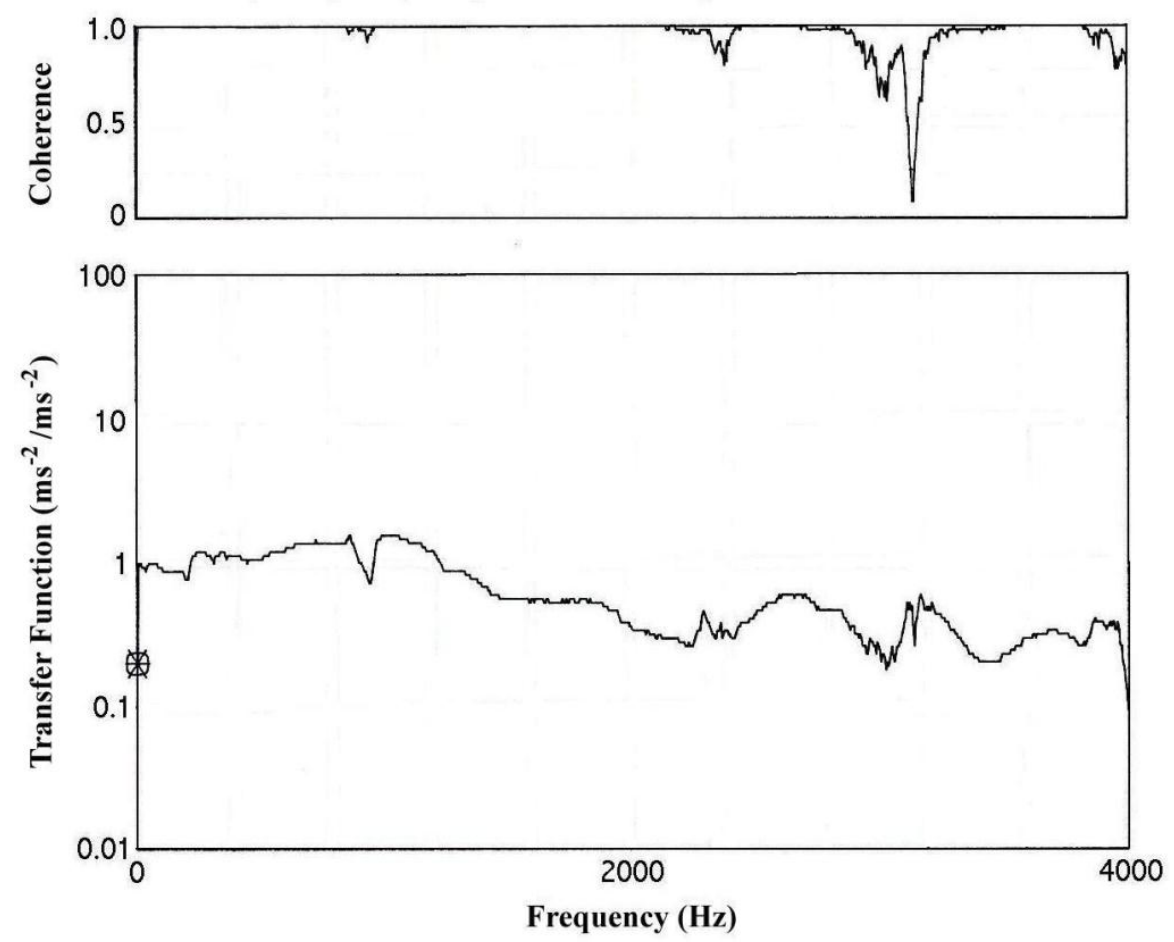

b) Frequency response of the adaptor in the $z$-direction
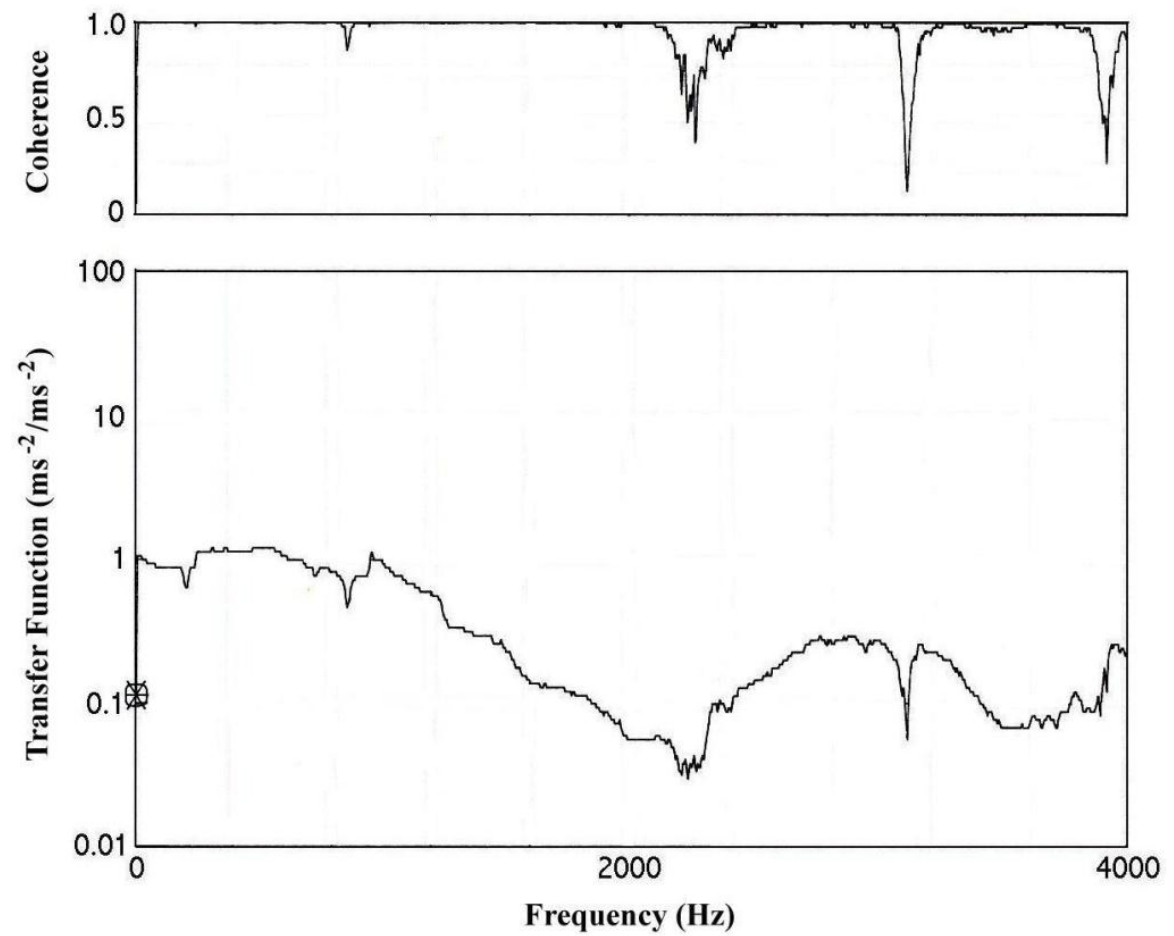

Figure 2. Frequency response of the adapter in each direction (lower part of each figure) and the coherence of the measurement (upper part of each figure).

long, $3 \mathrm{~mm}$ thick adapter was constructed from aluminium, had a mass of $17.6 \mathrm{~g}$ and it's frequency response in each measurement direction is illustrated in Figure 2. The upper graph, in both a) and b), shows the coherence of the measurement, whilst the lower graph shows that in each direction the frequency response of the adapter is acceptably flat up to approximately 1200 $\mathrm{Hz}$.

During the tests, the adapter was positioned beneath the left hand so that the accelerometers were $64 \mathrm{~mm}$ from the butt of the grip and aligned to measure vibration in the direction of strike (x-axis) and perpendicular to that and the shaft axis (z-axis), as illustrated in Figure 3; these 

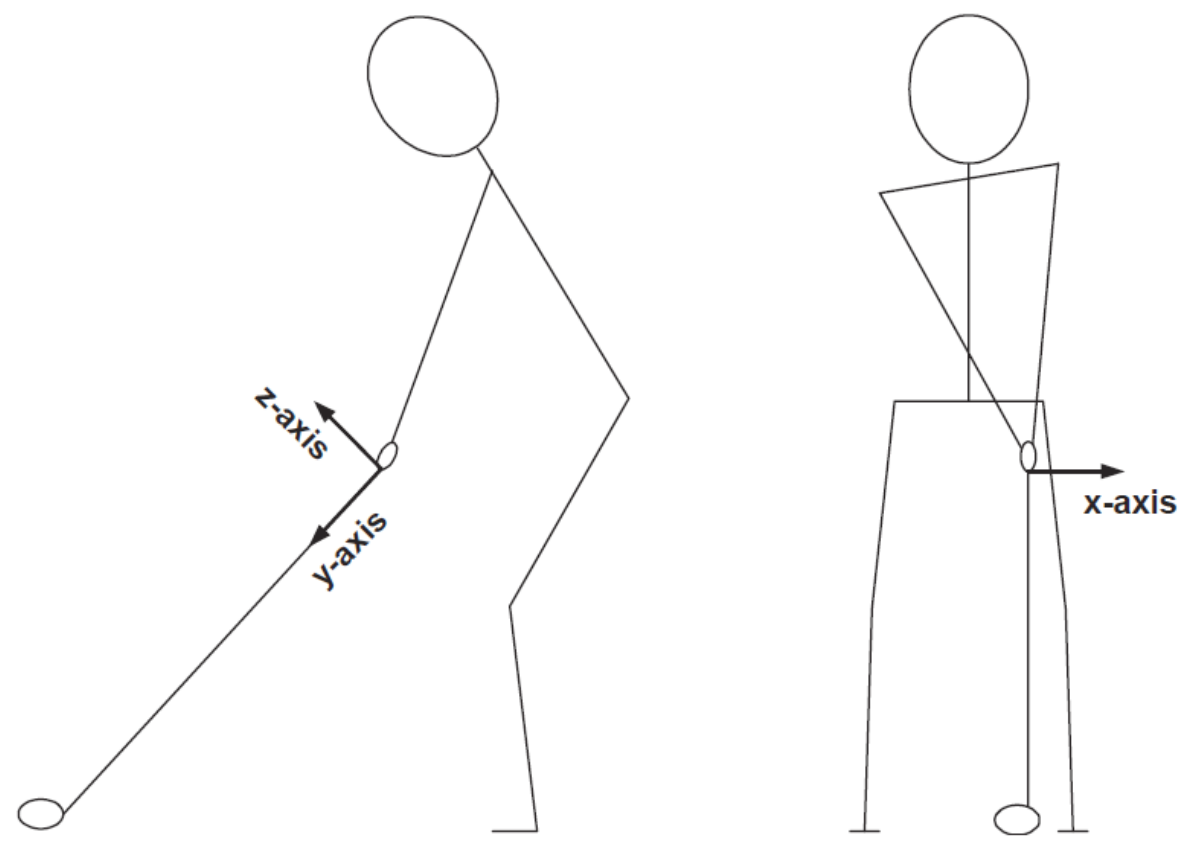

Figure 3. Vibration measurement directions.

measurements will subsequently be referred to as $x$-grip and z-grip. This measurement location was chosen because, for a right-handed golfer, there is a larger contact area between the left hand and the grip than the right hand and a previous study by Budney [11] reported the greatest grip forces immediately after impact to be produced by the left hand, possibly increasing vibration transmission to the hand.

Two more accelerometers were mounted on a second bracket clamped to the shaft $35.6 \mathrm{~cm}$ (14 inches) from the butt end of the grip, to measure shaft vibration in the same directions as the grip adapter, as illustrated in Figure $1 b$, and subsequently referred to as $x$-shaft and $z$ shaft. The purpose of these measurements was to examine how strongly shaft measurements could be correlated with golfers' responses. If good correlations could be obtained, this would justify discarding the less convenient grip measurements in future studies.

The cables from each accelerometer were run along the left arm, over the shoulder and down the back of each golfer and were held in place by a combination of wristbands and clips. A multi-channel data acquisition system was used to sample each signal at 5120 samples/second for $100 \mathrm{~ms}$ and a $2 \mathrm{kHz}$ low-pass filter was used to prevent aliasing; vibrations in the frequency range $8 \mathrm{~Hz}$ to about $1000 \mathrm{~Hz}$ are considered to be the most significant in previous hand/arm vibration studies [12, 19]. Although the accelerometer calibrations were not rechecked after testing, all accelerometers were less than one year old and all had up-to-date calibration certificates at the time of the tests. At no stage during testing was there cause for concern that any of the accelerometer calibrations were drifting or that the accelerometers had become faulty. All measurements taken complied with the relevant standard [19].

\subsection{Procedure}

Fifteen elite golfers, aged between 20 and 55 (mean 29, standard deviation 6 years) were selected. Two of the golfers were European Tour professionals, five were club professionals, one an assistant professional and seven were amateur golfers with handicaps between plus two and two. One of the European Tour professionals was female; the other golfers were all male. Ideally, duplicate tests would have been conducted with each subject to verify the repeatability of the results. The elite golfers used in this study, however, volunteered their time free of charge and it was not possible for them to participate in repeat tests soon after.

Ten clubs were selected, including modern titanium clubheads and older clubs with heads made of steel, laminated wood and persimmon, to cover a broad range of variations in feel. A three-piece wound ball was used for every test.

All fifteen tests were conducted in an indoor player testing facility. On arrival, the test procedure was outlined to each golfer. The five questions to be answered after each shot were described and also written up on a wallboard to increase the rate of data collection. The golfers were asked to avoid allowing preconceptions to affect their ratings. They were asked to rate each shot on its own merit, before looking at the impact location.

Club order was randomised according to a Latin square; each club featured once in each test and, over ten tests, in a different position in the order. During the tests, the golfers hit five shots with each of the ten clubs. All subjective and objective measurements were recorded for each shot played. In order to isolate vibration transmitted to the hands as the sole form of feedback from impact, the tests were conducted in a net and pink noise was 
played to the golfers through headphones to mask the sound of the impact. None of the golfers reported being adversely affected by the noise.

\section{Results}

3.1 Correlation of subjective data

Initially, the subjective ratings of 'pleasantness', 'hardness', 'vibration level', 'solidity' and 'liveliness' were correlated with each other to investigate relationships between the feel characteristics themselves. The Pearson method was used to measure the linear relationships between the ratings and correlation coefficients were calculated for each combination of feel characteristics for each of the fifteen golfers.

In general, very strong, positive correlations were found between pleasantness, solidity and liveliness of feel. For correlations involving the hardness of feel, the magnitude of the coefficients varied much more between golfers. In particular, three golfers' ratings of hardness of feel contradicted the general trend. Follow-up discussions with these golfers revealed that the question had been misinterpreted and, as a result, their hardness ratings were removed from the analysis.

To obtain an overall correlation coefficient for each combination of feel characteristics, all of the golfers' ratings needed to be combined but, in common with any tests involving rating scales, the golfers each instinctively used different ranges within the bounds of the scale. Following standard procedures to overcome such problems, the data was normalized [20] by taking each golfer's responses, subtracting that golfer's mean rating for that characteristic and then dividing by their standard deviation. Thus, for each feel characteristic, the normalized ratings for each golfer had a mean of zero and a standard deviation of one.

Combining all the normalized data and calculating the correlation coefficients gave the values shown in Table 2 . It can be seen that strong positive correlations are found within a group of characteristics containing pleasantness, solidity and liveliness of feel and also within a second group containing hardness of feel and perceived vibration level. Between the two groups, though, a negative correlation exists, so a shot that is rated as pleasant overall will also tend to be rated as feeling solid, lively, soft and with little vibration perceived.

\subsection{Correlation of subjective and objective data}

Initial inspection of the vibration data revealed a number of notable points. Vibration levels measured at the shaft were greater than vibration levels measured at the grip. In addition, vibrations measured in the $x$ direction, aligned to the direction of strike, were greater than the $z$-direction measurements at both shaft and grip. This can be seen in the example measurements in Figure 4. Low frequency content, due to the whole-body acceleration of the club during the swing has a noticeable effect on the z-grip data. For all measurements, the mean offset was subtracted prior to analysing the data.

\subsubsection{Selection of Vibration Parameters}

In order to correlate the subjective and objective data, peak-to-peak and r.m.s. levels were initially calculated for all four measurements from each shot. It is doubtful whether the golfers were able to distinguish the direction of vibration and so, in addition, the two shaft measurements and the two grip measurements for each shot were combined and the total r.m.s. level calculated at both the shaft and the grip. In general, it was found that the strongest correlations were obtained with the total r.m.s. vibration level, so the analysis continued using only this parameter. In the sections that follow, this total r.m.s. vibration level is abbreviated for convenience to 'r.m.s. level'.

Mean r.m.s. levels measured at the grip for central impacts with each club are shown in Figure 5 to illustrate the differences between clubs. The highest vibration levels occurred with clubs 3 and 7 , which are the two traditional wooden-headed clubs, whilst clubs 9 and 10, two of the modern titanium clubheads, generated only half the r.m.s. levels of the wooden-headed clubs.

\subsubsection{Data Normalization}

When the raw data from all the golfers were combined and the Pearson coefficients calculated between the subjective data and the vibration data, only weak correlations were obtained, as illustrated in the first two columns of Table 3. Despite their relatively small magnitude, the coefficients are statistically significant; with so much data, between 494 and 650 values depending on the feel characteristic, a Pearson coefficient with a magnitude of just 0.08 or greater is significant at

Table 2. Pearson correlation coefficients for each combination of normalised ratings ( $\rho$ value< $<$ o.oor for each coefficient).

\begin{tabular}{ccccc}
\hline & $\begin{array}{c}\text { Unpleasant - } \\
\text { Pleasant Feel }\end{array}$ & $\begin{array}{c}\text { Soft - Hard } \\
\text { Feel }\end{array}$ & $\begin{array}{c}\text { No Vibration - } \\
\text { Lots of Vibration }\end{array}$ & $\begin{array}{c}\text { Not Solid - } \\
\text { Very Solid Feel }\end{array}$ \\
\hline $\begin{array}{c}\text { Dead - Lively Feel } \\
\text { Not Solid - Very } \\
\begin{array}{c}\text { Solid Feel } \\
\text { No Vibration -Lots } \\
\text { of Vibration }\end{array}\end{array}$ - $^{\text {Soft - Hard Feel }}$ & -0.827 & -0.592 & -0.654 & 0.870 \\
\hline
\end{tabular}



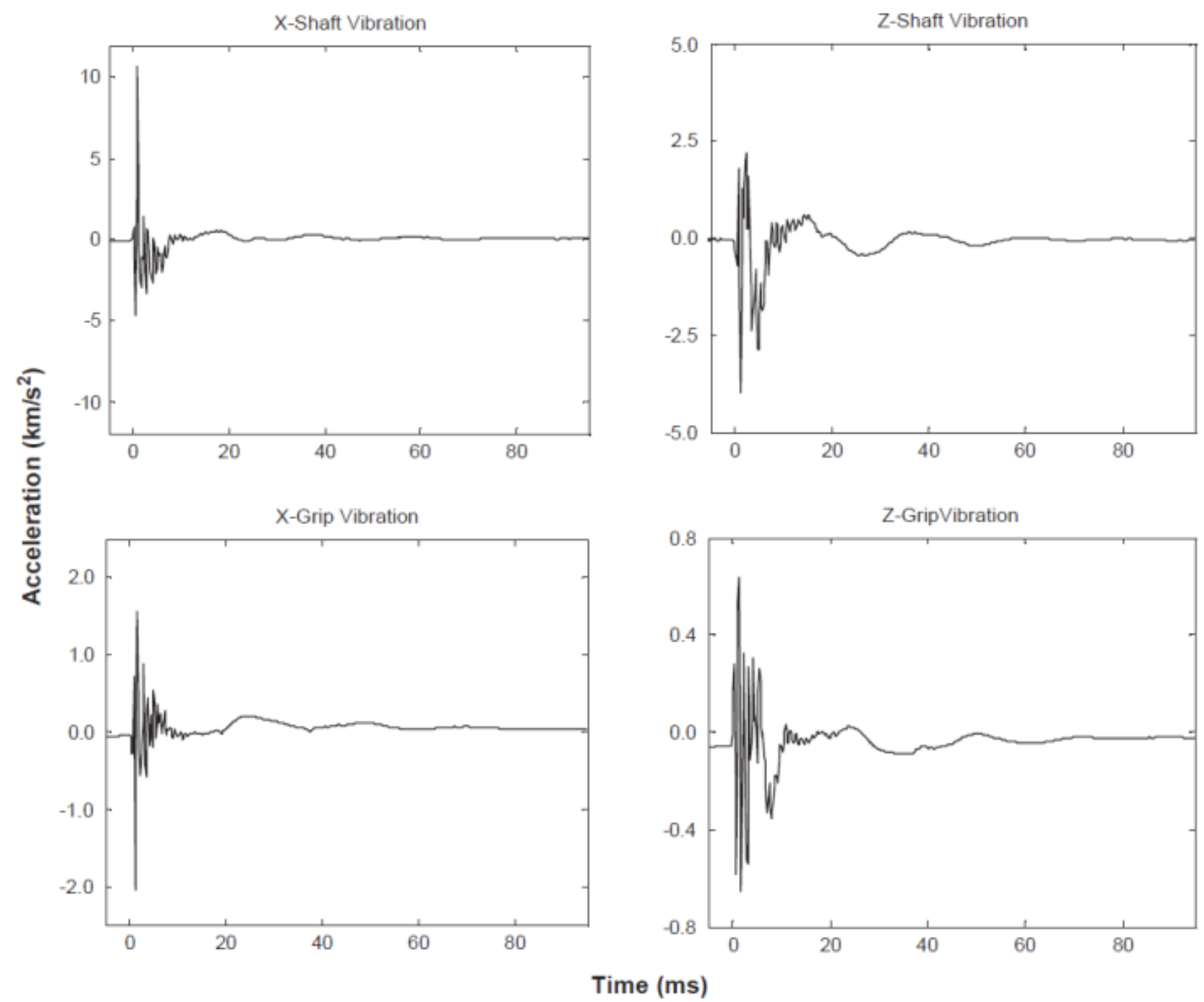

Figure 4. Example vibration measurements at both shaft and grip in the $x$ and $z$ directions.

the 0.05 level.

As already discussed, golfers vary in their use of the rating scales, so ratings were normalized to overcome this problem. It also appears that golfers rate the perceived vibration relative to their own reference level. Figure 6 shows the r.m.s. level for each golfer as a mean, \pm one standard deviation, calculated from all unweighted measurements at the grip with every club, to indicate the way in which each golfer's reference level may vary. Only the data from central impacts, within $10 \mathrm{~mm}$ of the geometric centre of the clubface, were used to calculate the mean values so that the data would not be influenced by the consistency and accuracy of the golfer. This graph indicates that there are variations in the mean grip vibration levels generated by each golfer from central impacts by as much as a factor of $2 \frac{1}{2}$. As a result, Subjects 9 and 10 might be expected to rate an r.m.s. level of 250 $\mathrm{m} / \mathrm{s} 2$ much lower than Subjects 4 and 5 . These variations are likely to be a result of differences in swing profiles, variations in clubhead speed at impact and differences in the grip of each individual golfer.
The vibration data was, therefore, normalized by subtracting, for each golfer, their mean r.m.s. level from the r.m.s. level of each shot they played. This increased the magnitude of the correlation coefficients by on average approximately o.1.

\subsubsection{Frequency Weightings}

Human response to vibration transmitted to the hand is frequency dependent [13] and consequently, ISO [18] (equivalent ANSI [21]) and BS [19] standards specify frequency weightings. The weightings, illustrated in Figure 7, are similar except below $10 \mathrm{~Hz}$; the BS weighting reduces to approximately o while the ISO weighting remains constant at 1 . For this study, the difference between the weightings is insignificant as the resolution of the vibration spectra is $10 \mathrm{~Hz}$. Consequently, only the BS weighting was used.

Pearson correlation coefficients were calculated for the frequency weighted vibration data and the subjective data. When compared to the coefficients obtained using 


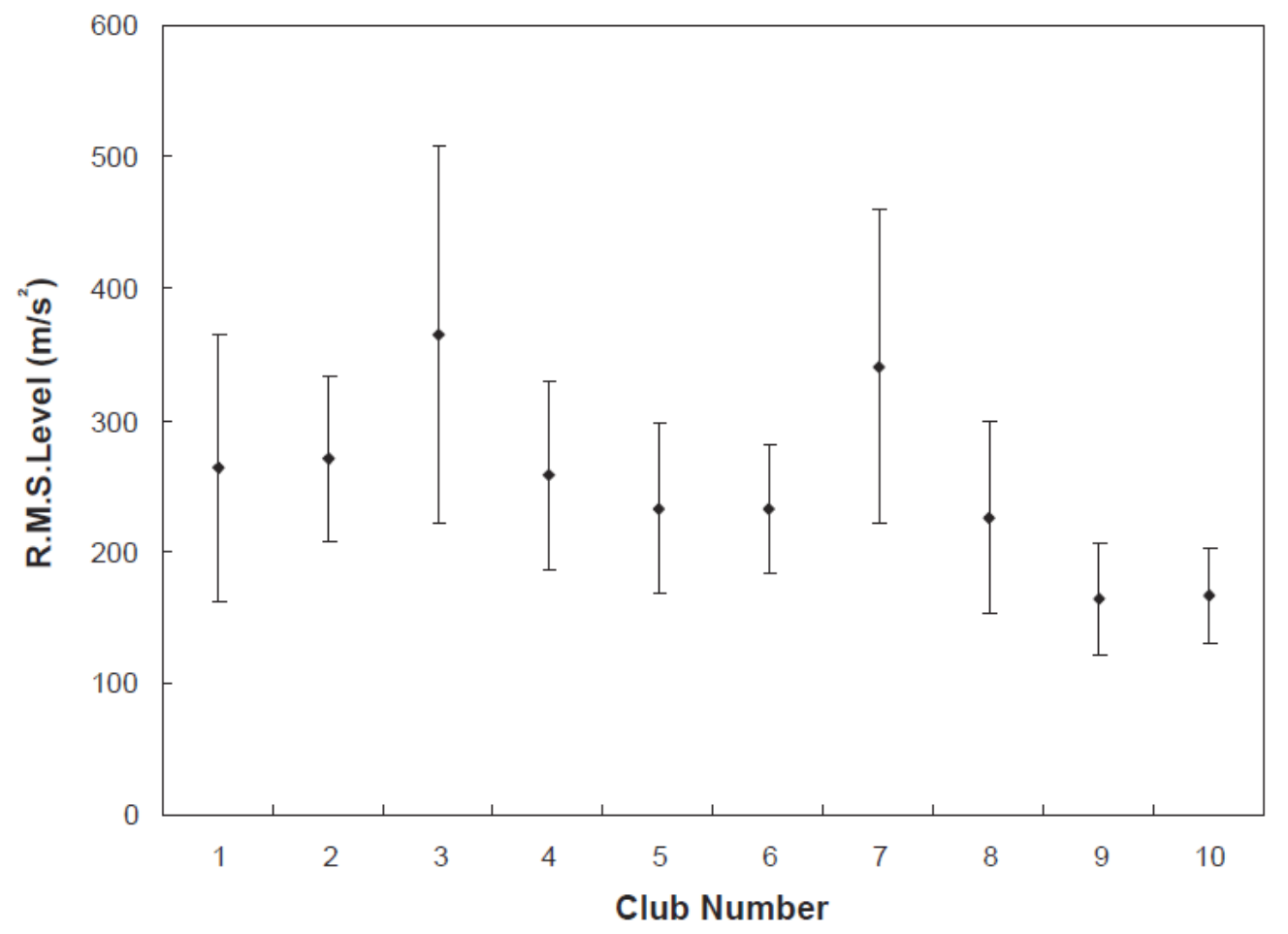

Figure 5. Mean unweighted total rms vibration level at the grip, \pm one standard deviation, of central impacts for each club.

unweighted data, the BS weighting was found to have increased the magnitude of the coefficients with shaft measurements by, on average, 0.04 but decreased them with grip measurements by o.1. This reduction for grip measurements was not expected and may be due to the contribution of low frequency swing accelerations in the measurements. As noted previously, the magnitude of grip vibration is considerably smaller than that at the shaft and therefore the effect of whole-body club acceleration is much greater. The BS weighting emphasises the low frequency content of a spectrum and, therefore, the swing acceleration will contribute more significantly to the weighted r.m.s. level of the grip than the shaft.

\subsubsection{Summary}

The best overall set of correlations with shaft measurements was obtained when the subjective ratings were normalized, the $x$ and $z$ vibration data BS weighted and the total r.m.s. levels calculated and normalized by subtracting the mean r.m.s. level. The best overall set of correlations with grip measurements was obtained using the same procedure except that the vibration data was not weighted. These results are summarised in the middle two columns of Table 3 .

When these values are compared to the coefficients calculated using the unprocessed data, shown in the first two columns of Table 3, it can be seen that frequency weighting the data, in the case of the shaft measurements, and normalizing has generally increased the magnitude of the coefficients by more than o.2. In contrast, however, the original ratings of hardness of feel correlate more strongly with unprocessed vibration data.

Other trends can also be observed. Pleasant feel, solid feel and liveliness of feel all consistently correlate negatively with r.m.s. level whilst hardness of feel and perceived vibration level correlate positively, implying a pleasant, soft, solid, lively feel is associated with a lower r.m.s. level.

The normalized BS weighted r.m.s. levels at the shaft and the normalized ratings of solid feel will be used to illustrate a typical correlation. The normalized ratings of solid feel from -3 to 2 were divided into ten bands of equal width. The mean and standard deviation of the normalized r.m.s. levels were calculated for the data in each band. The results, along with a linear best fit through the mean values, are plotted in Figure 8, which illustrates the negative correlation of -0.4 that exists between normalized r.m.s. level and solid feel. The graph also indicates that an increase in r.m.s. level of $20 \mathrm{~m} / \mathrm{s} 2$ above the golfers' mean values, which ranged from 100 to $200 \mathrm{~m} / \mathrm{s} 2$, resulted in their ratings decreasing by two standard deviations, typically between 2 to 3 points on the 9 point rating scales in Table 1.

\subsection{Correlations with mean data}

The task of reliably and repeatedly rating the feel of so many shots is difficult and the majority of golfers were inexperienced in participating in tests such as these. They were also being asked to hit shots in an unfamiliar manner; their hearing was restricted and they could not see the ball flight because the tests were conducted in a 
Table 3. Pearson correlation coefficients for each combination of feel characteristic and vibration parameter using different data processing techniques (all coefficients are significant at the 0.05 level)

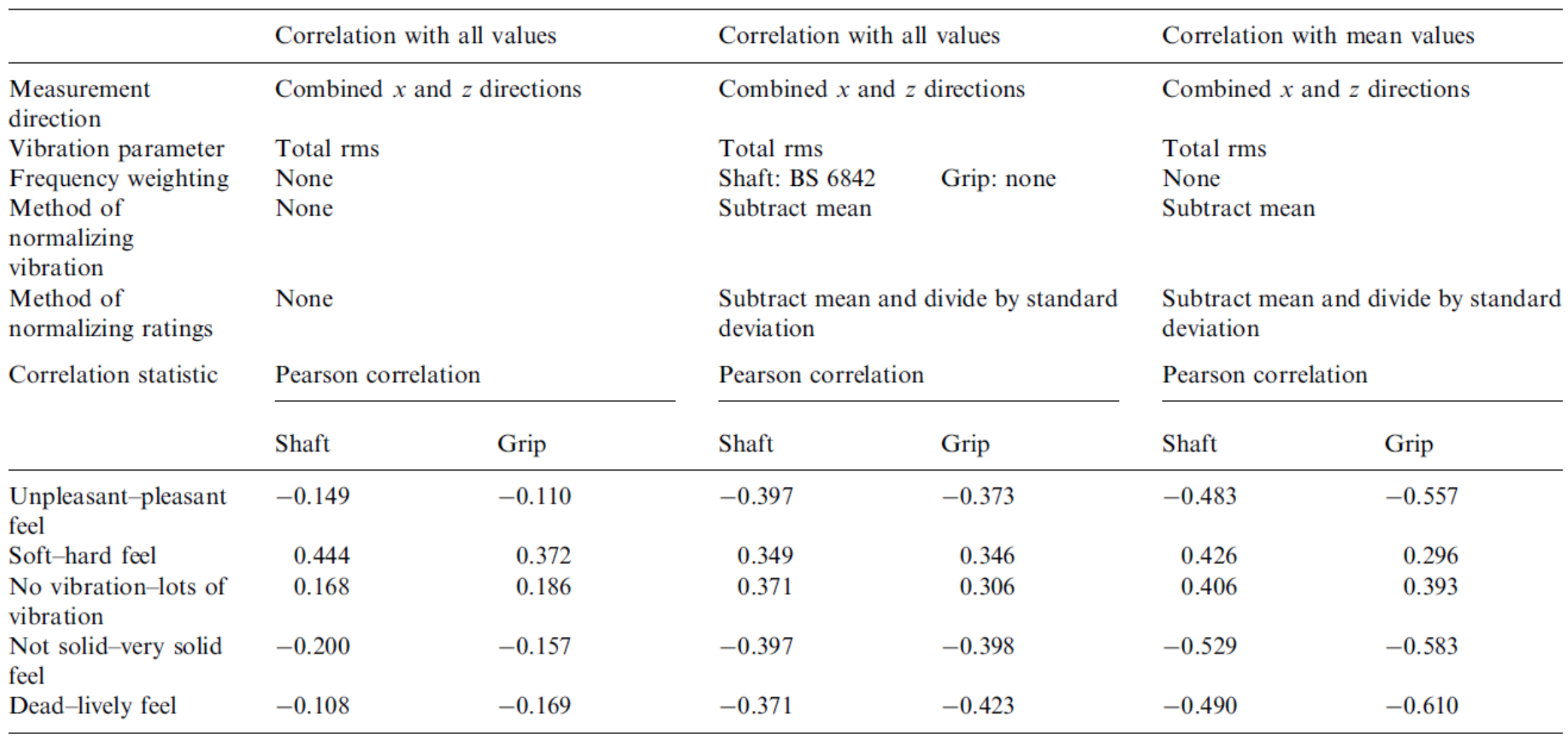




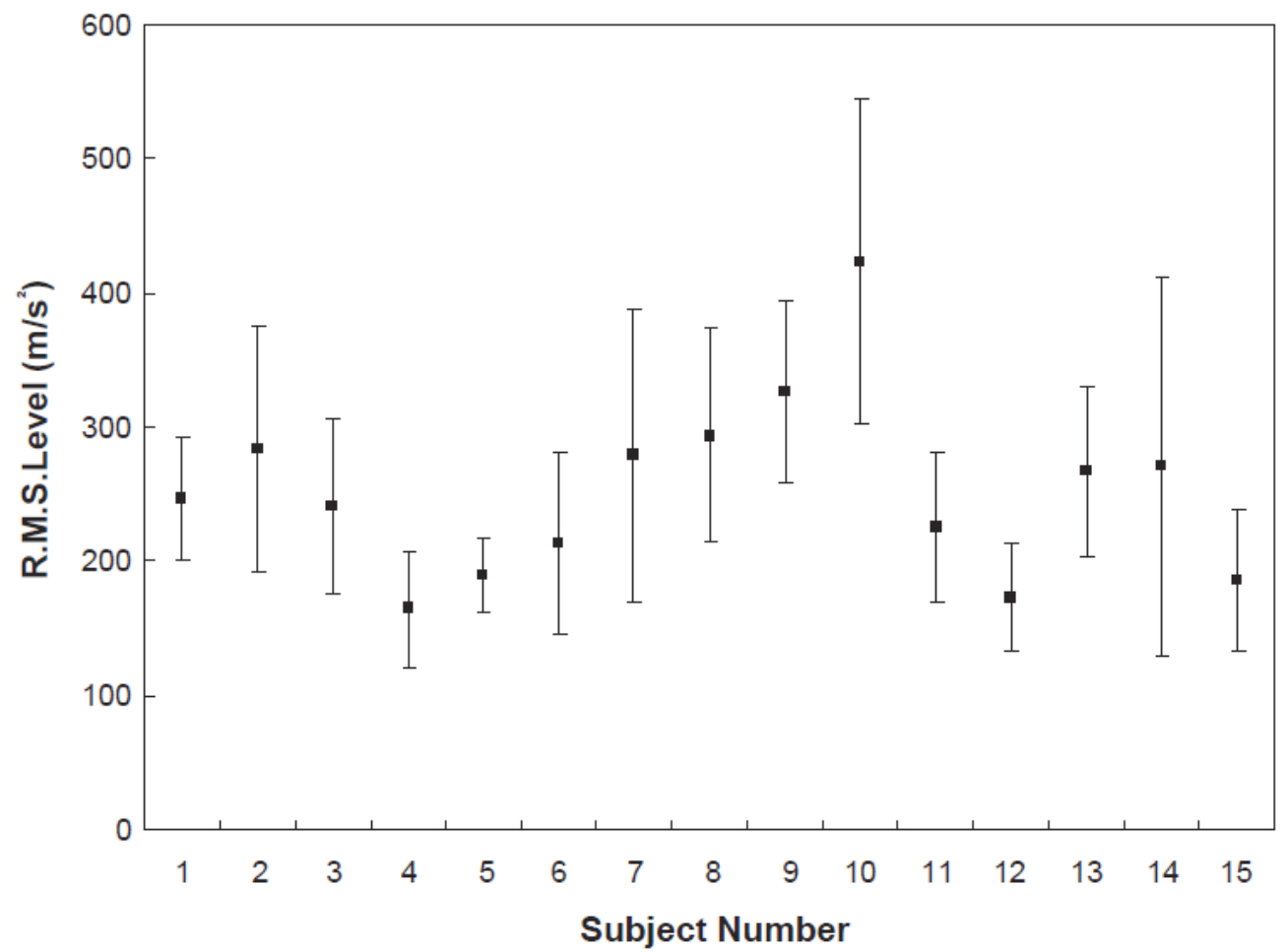

Figure 6. Mean unweighted total rms vibration level at the grip, \pm one standard deviation, of central impacts for each golfer.

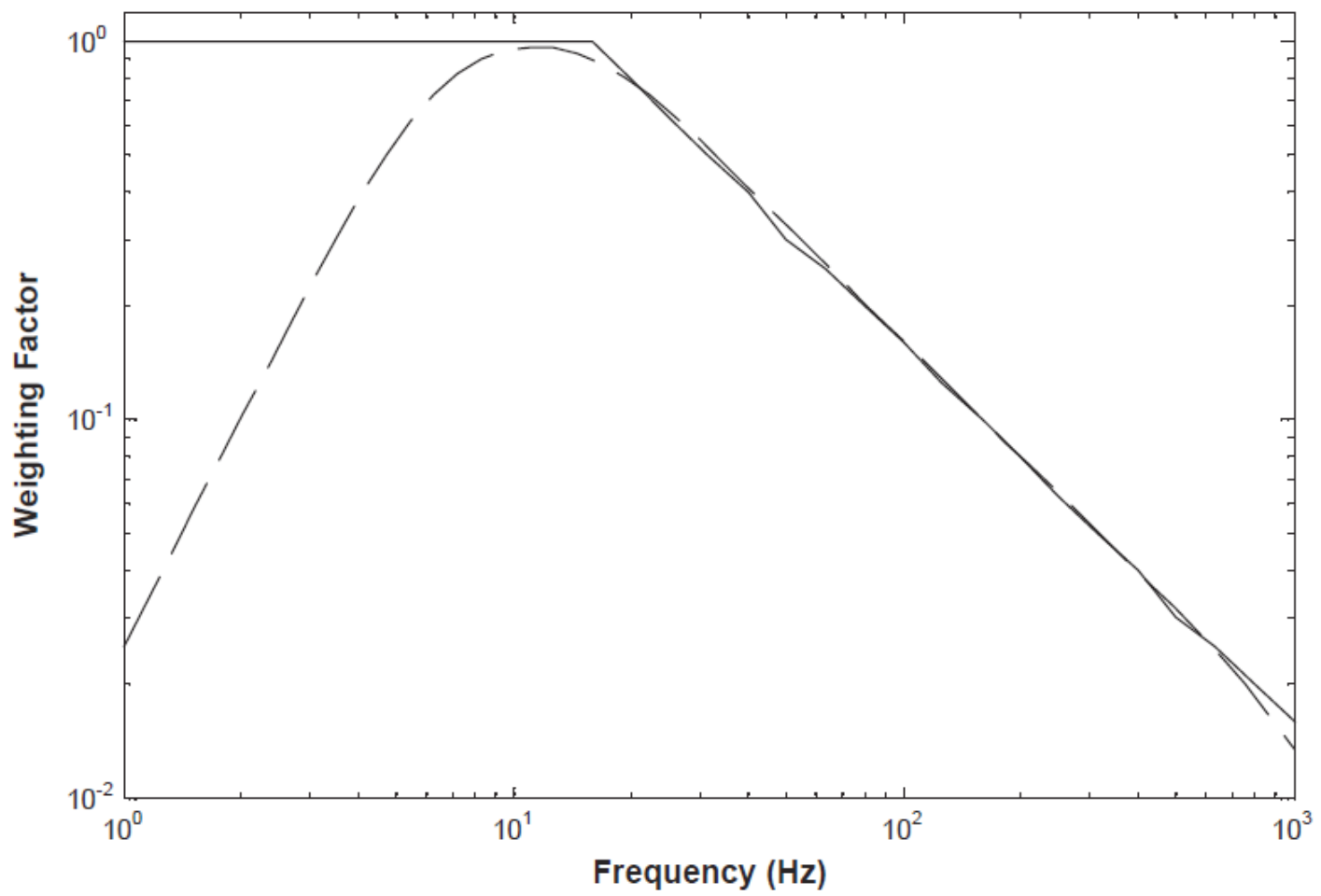

Figure 7. BS(- - -) and ISO (-) frequency weightings for hand-arm vibration 


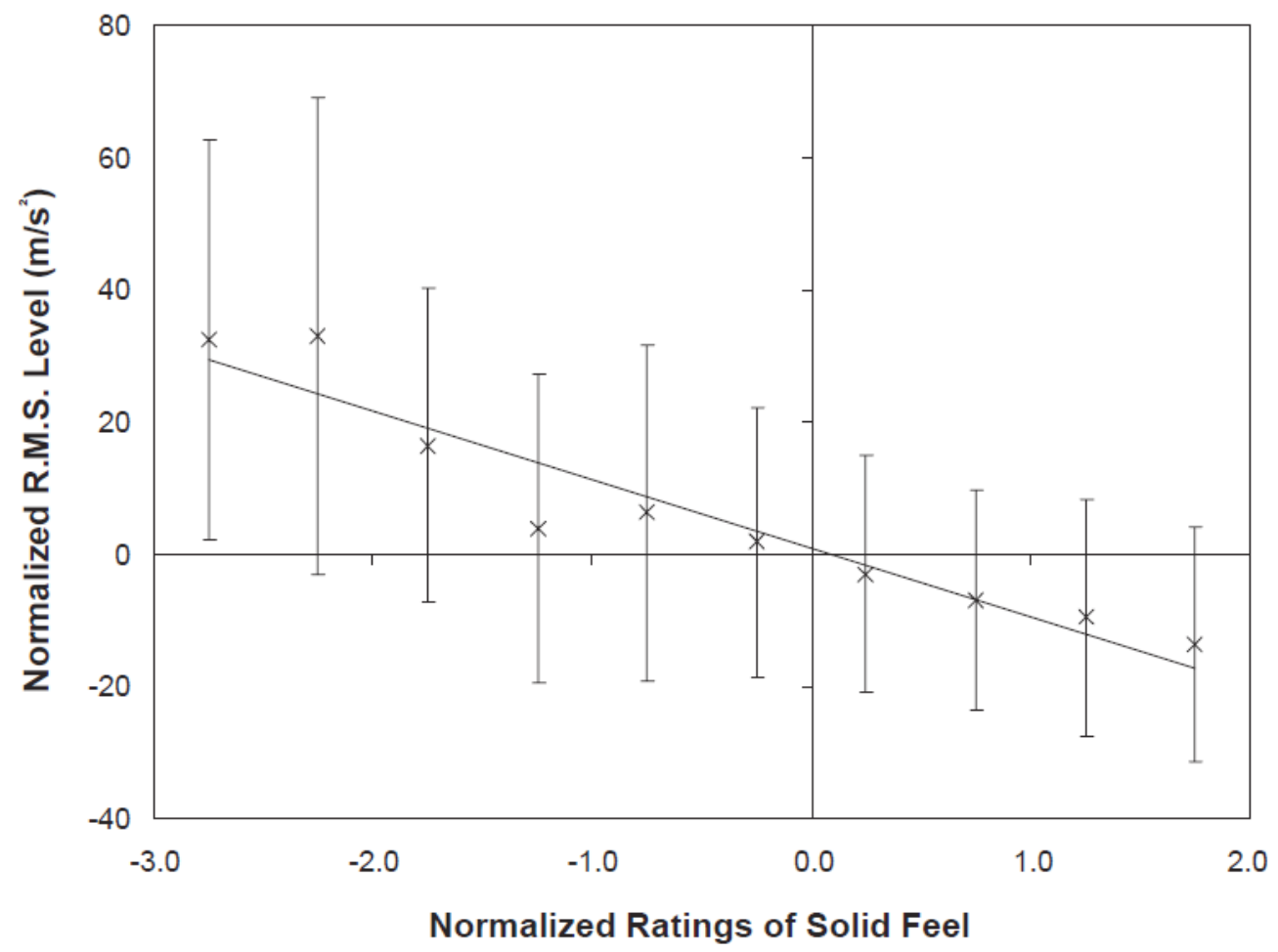

Figure 8. Correlation between normalised measurements of BS weighted total rms vibration level at the shaft and solid feel.

net. For these reasons, it is possible to obtain individual data points far away from the genuine trend. The effect of these values on the correlations would be minimised by calculating, for each golfer, a single mean rating for each question and mean value for each vibration parameter from the five shots with each club. Correlation coefficients were then recalculated using these mean values rather than the values from individual shots.

Using the mean data, the strongest overall set of correlations was obtained for both shaft and grip measurements using normalized subjective ratings and normalized, unweighted r.m.s. levels. The coefficients obtained are listed in the last two columns of Table 3; with approximately 135 values being correlated, a coefficient greater than 0.166 in magnitude is significant at the 0.05 level.

Compared with the coefficients in the middle two columns, the results show that the strength of the correlation has generally been improved by using mean values for each club. These improvements are only achieved, however, with the unweighted vibration data.

The considerably larger values of the Pearson coefficients obtained using the mean values for each club and for each golfer suggest that this technique is the most suitable for analysing the data obtained in these tests.

\section{Conclusions}

This study was conducted to investigate correlations between golfers' subjective perceptions of the feel of a shot and the vibration measured from impact. Tests, representative of actual play conditions, were designed such that both subjective and objective data were measured simultaneously from each shot.

Analysis of the subjective ratings revealed that the five feel characteristics investigated - pleasantness, hardness, solidity, liveliness and perceived vibration level - were strongly correlated. In general, shots that were given a high pleasantness rating were also rated as having felt solid, lively, soft and with little vibration perceived. When relationships between the raw subjective and objective data were investigated, the correlations were weak. Normalizing both the subjective ratings and the objective data was particularly successful in improving the strength of the correlations. Calculating the total r.m.s. vibration level at both shaft and grip achieved useful but smaller enhancements while the application of the BS frequency weighting was largely unsuccessful and there is little evidence from this study to suggest its suitability for similar future studies. The strongest correlations were obtained when the mean data for each club for each golfer were correlated. These relationships suggest that a shot with a lower total r.m.s. vibration level has a pleasant, very solid, lively and softer feel.

The magnitudes of the correlation coefficients obtained in this study have tended to vary between 0.3 and 0.6 and, although statistically significant, it is believed that stronger correlations could be achieved. From the experience gained conducting this and other tests, a number of possible improvements to the test procedures have emerged. The identification of reliable test subjects, based on the consistency of their ratings during testing, and the use of mean data based on several shots rather than individual shots will be investigated. Both factors 
point towards the use of paired comparison methods and these will be explored in future testing.

\section{Acknowledgements}

The authors would like to thank the golfers without whose time and effort this study could not have been conducted.

\section{References}

1. A. Cochran and J. Stobbs, The Search For The Perfect Swing, Heinemann, London, 1968.

2. M. Hedrick and M. Twigg, The Feel Of A Golf Shot: Can We Measure It?, in: A. Cochran (Ed.), Golf: The Scientific Way, Aston Publishing Group, Hemel Hempstead, UK, 1994, pp.131-133.

3. A. Hocknell, R. Jones and S. J. Rothberg, Engineering 'feel' in the design of golf clubs, in: S. J. Haake (Ed.), The Engineering of Sport: Proceedings of the 1st International Conference on the Engineering of Sport, A.A.Balkema, Rotterdam, NL, 1996.

4. T. E. Fairley, Some Measurements of Tennis Racket Vibration and its Transmission to the Hand, in: United Kingdom Informal Group Meeting on Human Response to Vibration, Derby, UK, 1985.

5. E. M. Hennig, D. Rosenbaum and T. L. Milani, Transfer of Tennis Racket Vibrations onto the Human Forearm. Medicine and Science in Sport and Exercise 24 (10) (1992) pp.1134-1140.

6. P. S. Varoto and K. G. McConnell, Using Modal Analysis to Evaluate Golf Club Performance. Sound and Vibration 29 (3) (1995) pp.20-23.

7. R. C. Merkel and T. Blough, Dynamic Characterization and Comparison of Golf Clubs, in: Proceedings of the 17th International Modal Analysis Conference, FL, USA, 1999, pp.513517 .

8. A. L. Wicks, C. E. Knight, P. Braunwart and J. Neighbors, The Dynamics of a Golf Club, in: Proceedings of the 17th International Modal Analysis Conference, Fl, USA, 1999, pp.503-508.

9. J. S. B. Mather and S. Jowett, The effect of centrifugal stiffening on the bending stiffness of a golf shaft, in: S. J. Haake (Ed.), The Engineering of Sport: Proceedings of the 2nd International Conference on the Engineering of Sport, Blackwell Science, Oxford, UK, 1998.

10. G. Thomas, T. Deiters and C. Best, Simulating Golf Club Performance using Modal Analysis, in: Proceedings of the 13th International Modal Analysis Conference Vol. 2, Society for Experimental Mechanics, 1995.

11. D. R. Budney, Measuring Grip Pressure During the Golf Swing. Research Quarterly 50 (2) (1979) pp.272-277.

12. M. J. Griffin, Handbook of Human Vibration, Academic Press, London, 1990.

13. D. D. Reynolds, K. G. Standlee and E. N. Angevine, Hand-Arm Vibration, Part 3: Subjective Response Characteristics of Individuals to Hand-Induced Vibration. Journal of Sound and Vibration 51 (2) (1977) pp.267-282.

14. T. Miwa, Evaluation Methods for Vibration Effect: Part 3. Measurements of Threshold and Equal Sensation Contours on Hand for Vertical and Horizontal Sinusoidal Vibrations. Industrial Health 5 (1967) pp.213-220.

15. L. Noble and H. Walker, Baseball Bat Inertial and Vibrational Characteristics and Discomfort Following Ball-Bat Impacts. Journal of Applied Biomechanics 10 (1994) pp.132-144.

16. C. L. Stroede, L. Noble and H. S. Walker, The effect of tennis racket string vibration dampers on racket handle vibrations and discomfort following impacts. Journal of Sports Sciences 17 (1999) pp.379-385.
17. J. Roberts, R. Jones, C. Harwood, S. Mitchell and S. Rothberg, Human Perceptions of Sports Equipment under Playing Conditions. Journal of Sports Sciences 19 (7) (2001) pp.485-497.

18. ISO 5349:1986, Mechanical Vibration - Guidelines for the measurement and the assessment of human exposure to hand-transmitted vibration.

19. BS 6842:1987, Measurement and evaluation of human exposure to vibration transmitted to the hand.

20. N. J. Mansfield, P. Holmlund and R. Lundström, Comparison of subjective responses to vibration and shock with standard analysis methods and absorbed power. Journal of Sound and Vibration 230 (3) (2000) pp.477-491.

21. ANSI S3.34-1986, Guide for the measurement and evaluation of human exposure to vibration transmitted to the hand. 
